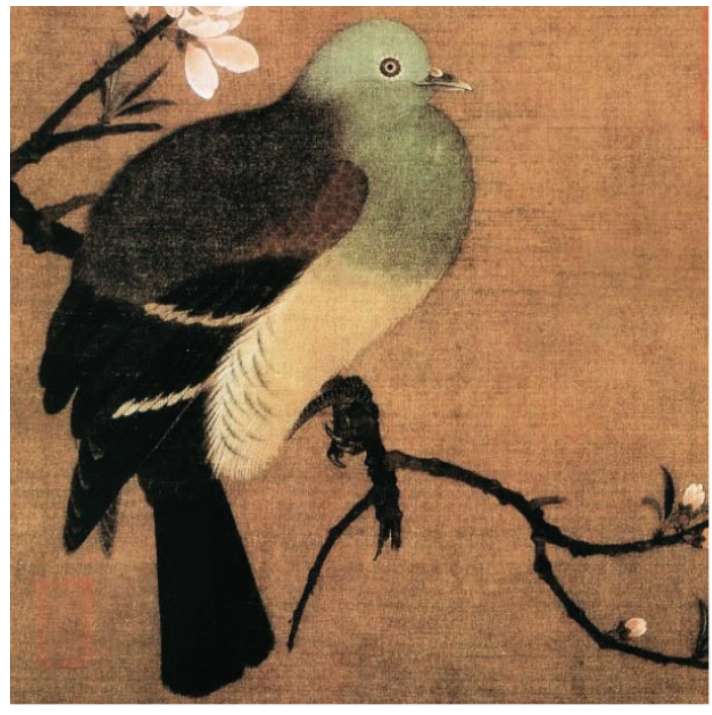

科 学

\section{Science and Art}

\section{你需要怎样的图片?}

浅浅阳光

美捷登图形与图像编辑

生物医学是可视化极强的学科。在基础医学 中, 我们通过大量视觉形象获取直观的医学感受,
例如组织切片、解剖标本、药用植物、细胞模型等; 在临床医学中, 视诊了解患者有无水肿、黄疸、 三凹征、蜘蛛㽽等直接带给医生患者的第一印象, 辅助检查中的心电图、超声、CT、核素等更是视 觉图像丰富的来源; 在医学研究中, 蛋白电泳、 免疫组化、电镜研究、计算机生物模型等都是视 觉化的输出结果; 在出版领域, 您发表论文时, 出版商需要你提供统计图表、流程图和各种形式 的示意图（图 1)。

在西方发达国家, 生物医学可视化工作已经 属于非常成熟的商业行为, 不仅高级医学院校、 大型出版集团有专人负责各种形式的生物医学可 视化项目, 例如图片、动画、视频等, 还有众多 的专门从事科学可视化的纯商业公司, 例如图片 社、医学插画公司、科学图片服务商等。由于这 些国家拥有过成熟的版权法, 通过商业行为制作 的可视化材料都是版权材料, 价格不菲, 一张授 权的版权图片报价在 500 元以上是很常见的。

目前在国内也有一些零星的团队、公司和个 人在从事商业化的图片服务工作，但受很多因素

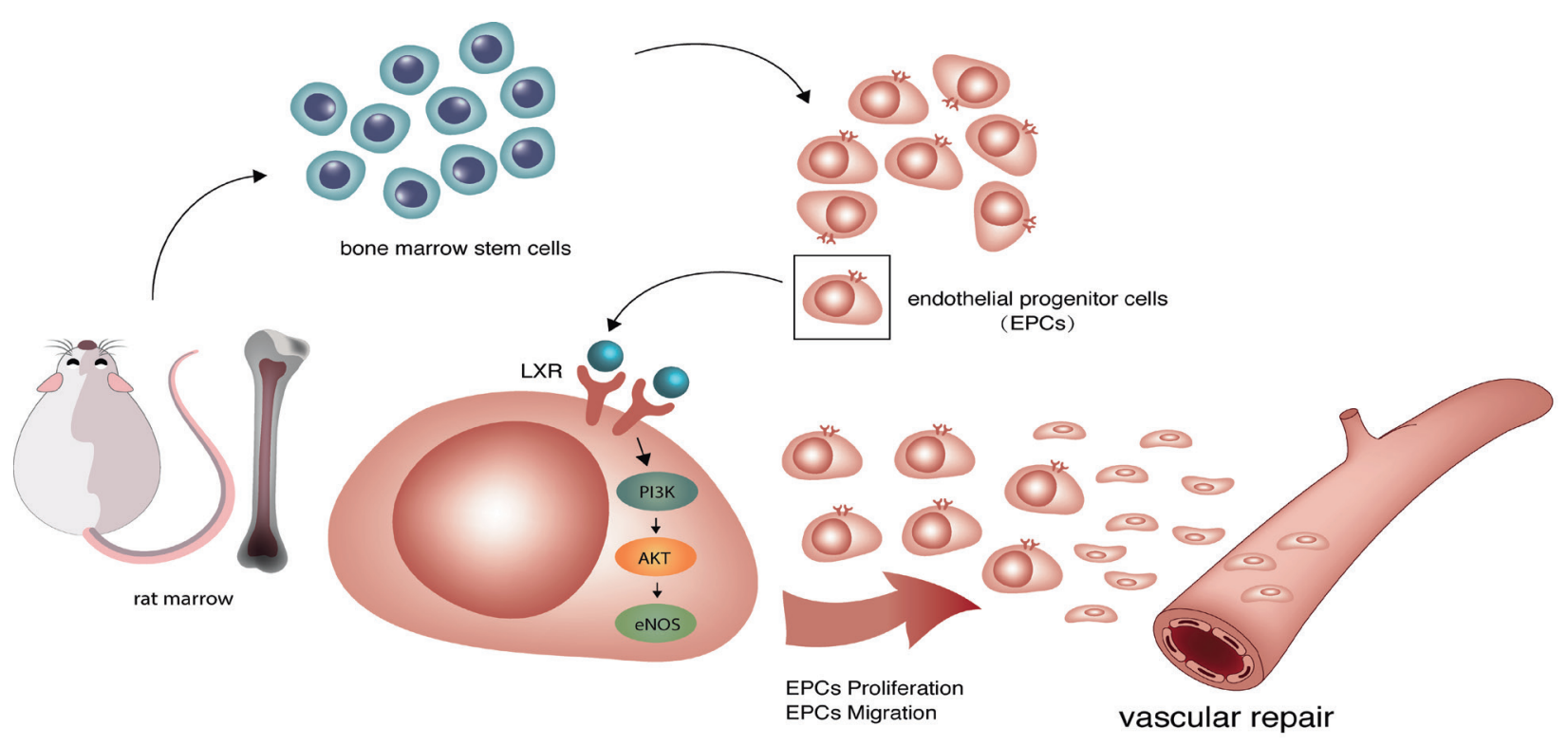

图 1 笔者为某医科大学教授的论文绘制的原创医学插画 
限制, 与国外相比, 商业化进程差距很大。这就 造成了两个局面: 一方面众多需要图片等可视化 服务的科研人员、作者、出版社找不到专人制作; 另一方面, 从事科学可视化服务的人员接不到相 应的项目, 处于 “吃不饱” 的状态。可喜的是, 美捷登公司意识到这一点, 率先在国内开展了生 物医学图片以及可视化服务, 极大的方便了国内 的生物医学研究人员。

一张精美的原创图片能够提高论文的原创动 力, 获得编辑和出版商的青睐。笔者当年为某医 科大学临床医学中心的一位博士绘制论文原创医 学插画时, 审稿编辑来信回复该博士:

"Nice report of interesting approach to cervical spine pathology. Well done and a nice report. The images and drawings help to clarify the text even more."

很快回复作者论文录用。国外出版商和编辑 非常喜欢原创动力的研究论文, 除了研究本身的 独创性、新颖性以外, 原创医学插画也是提高论 文原创动力的一部分, 因为一幅精心设计的原创 医学插画有利于提高论文的引用频次, 原创图片 能广泛用于课件的制作, 有利于论文和刊物的推 广, 扩大影响力, 甚至能被其他作者再次引用出版, 而二次出版需要为原出版商支付不菲的图片授权 使用费, 这种二次出版在生物医学出版领域是非 常多见的, 例如你需要对方的图片来阐明自己的 某些观点, 编写教科书的需要, 制作动画的需要等。

当你需要为你的论文配置原创医学插画时, 要有的放矢, 应是你论文中最重要、最复杂、最 独创性的观点或文字配置原创医学插画, 而不是 泛泛而论, 配置很多无关紧要的插图, 冲淡论文 主题。另一方面, 一些刊物的彩图印刷, 是要收 取昂贵的印刷费用的。

那么我们应该怎样为自己或设计师提出自己 的图片需求呢? 你需要掌握以下一些原则:

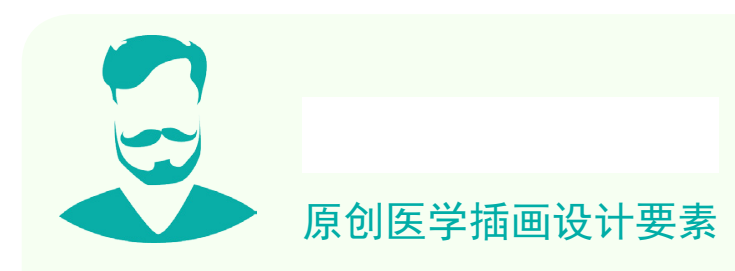

1. 画什么: 图片要表达怎样的内容呢? 是独创 的工艺流程, 还是复杂的医学知识 阐述等。设计师会根据你的内容选 择合适的制作工艺。

2. 对谁画: 是学术论文出版商, 还是图书出版 商; 是面向专业人士, 还是媒体, 还是普通公众呢? 设计师会根据你 对象, 设计不同艺术风格的图片。

3. 如何画: 设计师会倾听你的需求, 为你选择 合适的图片类型, 例如三维模型虽 然能更展示空间结构, 但有时二维 模型更容易让对方理解复杂的医学 经过。是矢量图更好呢, 还是位图 更好? 是黑白图更好呢, 还是彩图 更好等等。

4. 何处画: 设计师会询问你期待把图片用于何 处? 如果是学术论文出版商, 他们 会询问刊物名称，出版国家，甚至 亲自查询刊物已发表过的医学图 片, 帮助你绘制达到出版商要求的 合格图片。

5. 为何画: 当你需要原创医学插画时, 应告诉 设计师你的目标, 是满足普通发表 / 出版水平呢, 还是期望帮助论文 更上一层楼。设计师会据此帮你决 定图片的精细度、精美度, 提高图 片的原创动力。如果你的图片, 只 用于教科书的黑白印刷, 就完全没 有必要绘制彩色图片。

原创医学插画的完成需要作者和设计师通力 配合, 反复修改润色, 才能达到期望的效果。为 保证图片质量，最好根据你的时间，提前 1 月预约。 